\title{
Intra-Abdominal Pressure Monitoring
}

\author{
Zsolt Bodnar
}

Additional information is available at the end of the chapter

http://dx.doi.org/10.5772/intechopen.70701

\begin{abstract}
Pancreatitis, inflammatory processes or retroperitoneal haemorrhage, paralytic ileus, ascites, severe visceral oedema caused by extreme fluid replacement, blunt abdominal trauma, peritonitis, or even massive transfusion can be found among the triggering factors of intra-abdominal hypertension and abdominal compartment syndrome (ACS). The only possible way of establishing the diagnosis is to measure the intra-abdominal pressure (IAP), a widespread manner of which is the measurement through the bladder. In our works, we wanted to study whether the method of continuous intra-abdominal pressure monitoring is feasible within the everyday practice of diagnosing the conditions having increased intra-abdominal pressure. The globally accepted pressure measurement carried out through a urinary catheter and its classical so-called intermittent form has been employed worldwide in the intensive care units and surgical wards. The procedure is simple, yet time consuming, and the catheter connections and disconnections are sources of infection. The measurement results provide information only on the individual pressure values of the predetermined measurement dates. In order to eliminate these weaknesses and for the safe and quick measurements, the classical technique was replaced by a completely new method: the continuous intra-abdominal pressure monitoring. In order to determine the objectivity of the continuous intra-abdominal pressure measurement technique, we carried out a validation study on surgical patients with normal and elevated intra-abdominal pressures. The pressure was determined by both methods in case of all patients. Significant difference could not be observed between the results of the intermittent and of the new technique. In this chapter, we want to discuss in detail of this validation study appointing the strong advantages of the new monitoring process. Measurement of the intra-abdominal pressure is essential in the differential diagnosis of acute abdominal pathologies. Pressure measurement through urinary catheters for the monitoring of the intra-abdominal pressure, especially its continuous variant, is an excellently applicable method. Introduction into the daily clinical routine is highly recommended.
\end{abstract}

Keywords: intra-abdominal pressure, intermittent intra-abdominal pressure measurement, continuous intra-abdominal pressure monitoring, intra-abdominal hypertension, abdominal compartment syndrome 


\section{Introduction}

Human body is subdivided into smaller or larger units by well-defined compartments. The function of these compartments is to mechanically protect and separate from one another the organs or organ systems situated inside them. Distinctively separated spaces of our bodies are the different fascial compartments, the skull, the spinal canal, the orbit, the pericardium, and the thoracic and the abdominal cavities [1]. The elasticity of the tissues of the separating walls (bone, muscle, connective tissue) have a strong determinative effect on the tolerance for volume or pressure changes exerted on the organs, which can be found inside these compartments. Compartment syndrome in a wider sense defines those changes that occur in the given compartments due to the increased pressure (which apart from some lesser and/or greater fluctuations is constant under physiological circumstances) and to the decrease in local circulation developing in consequence of this. Detrimental effects of the increased pressure are widely known and precisely described in the medical literature [1-7]. Herniation syndromes occurring as a consequence of the increased intracranial pressure, the clinical appearance of pneumothorax and haemothorax caused by pathological accumulation of air or fluids inside the thoracic cavity, as well as the concept of pericardial tamponade are known by everybody. Although approaching these from this point of view is not routinish, yet no one questions that all the above cases represent a compartment syndrome occurring as a consequence of the increased pressure having been elevated due to certain specific reasons. Upon mentioning, associations are immediately made to fascial compartments; however, the term compartment syndrome means the clinical picture of the entirety of pathophysiological alterations developing in consequence of the increased pressure occurring within a closed space; and this is irrespective whether the separating compartment itself is formed by the skull, the thorax, the abdominal cavity, or a given fascial compartment. A common characteristic of these syndromes is the permanent and irreversible damage that may affect the organs, which can be found inside the given compartment if quick intervention cannot be provided. If vital organs are affected, these damages can be life-threatening or may even lead to death.

Abdominal compartment syndrome (ACS) was first described in relation to abdominal traumatic injuries, but its occurrence is not a bit scanty in the general surgical patient material, despite the fact that its aetiology is completely different [1,2]. Kron [8] was the first who albeit did not use the term itself, yet described compartment syndrome in 1984. It was again Kron who routinely used abdominal pressure measurement through bladder catheterisation [8], which became widespread by 1989; however, the fundamentals of the method were described 100 years prior by Oderbrecht. Later on, several research groups developed the method [9-13]. The creation of abdominal compartment syndrome as technical term is associated with the work of Fietsam et al in 1989. The golden age of ACS has been launched by the two papers of Schein and Burch published in 1995 and 1996, respectively.

Pancreatitis, inflammatory processes or retroperitoneal bleeding, paralytic ileus, ascites, and severe visceral oedema caused by extreme fluid replenishment, blunt abdominal trauma, peritonitis, or even massive transfusion can be found among the triggering factors of ACS; i.e., all factors that may and can lead to a sudden increase in the intra-abdominal pressure 
(IAP, 5-10 mmHg under physiological circumstances), to conditions of intra-abdominal hypertension (IAH, IAP $\geq 12 \mathrm{mmHg}$ ) associated with organ or multiple organs' failure, or without intervention to abdominal compartment syndrome (IAP $\geq 20 \mathrm{mmHg}$, which is associated with the failure of vital organs) $[2,7,11,12]$. Despite the modern and quick diagnostics and the adequate surgical interventions performed in time, the mortality of ACS is extremely high (38-71\%). It affects practically all vital organ systems such as cardiovascular, respiratory, urinary and central nervous systems, as well as the parenchymatous organs.

The only possible way of establishing the diagnosis is to measure the intra-abdominal pressure. A widespread manner of measurement is the method used through the bladder [8-11]. The fundamental principle of the method is the law which says that if pressure is exerted on the surface of a compartment predominantly containing some kind of fluid, then this pressure imposed upon the practically incompressible fluid will be transmitted unaltered to each and every point of the affected compartment. Consequently, the IAP and the intravesical pressure values are strictly identical. If the bladder is filled with $50 \mathrm{~mL}$ of physiological saline and the previously inserted catheter is closed, then the pressure predominating the bladder will be transmitted to the catheter and become easily measurable through a sterile needle inserted into the catheter. This procedure was simplified by the working group of Sugrue [11], who placed a 'T-element' into the catheter, which rendered unnecessary the closure and insertion of it, and also significantly reducing the prevalence of infections associated with this measurement. To surmount points of weakness (laboursome, intermittent), Balogh and his working group [13] developed and validated the method of continuous intra-abdominal pressure monitoring (CIAPM). Owing to their modifications, the procedure of vesical filling, catheter closure, and needle insertion was smoothed away ('Balogh-Sugrue technique').

Treatment of ACS is nearly always surgical decompression. Within the frame of prevention or in the case of individual ACS responding well to conservative methods, non-surgical solutions may also be possible (evacuation of the intraluminar content, removal of the space occupying process, improvement of the tolerance of the abdominal wall, optimal fluid therapy, optimisation of the systemic and regional circulation) [14-16]. Success is greatly influenced by the aetiology of the given case and by the general condition of the patient. If IAP $>20 \mathrm{mmHg}$ (and/ or APP $<50 \mathrm{mmHg}$, where APP = abdominal perfusion pressure) and new signs of organic dysfunction are occurring, then the ACS is not responding to the conservative method, and the possibility of surgical decompression should be carefully considered.

Surgical treatment for all cases of IAH/ACS is decompression laparotomy with temporary abdominal wall closure or open abdominal treatment. Following decompression, the problem cannot be regarded as solved, and the conservative method should be carried further on (adequate medication, optimal fluid therapy) along with the constant monitoring of IAP. APP above $60 \mathrm{mmHg}$ and IAP under $12 \mathrm{mmHg}$ mean the solution of the IAH. If APP $>60 \mathrm{mmHg}$ and IAP $>12 \mathrm{mmHg}$, then the conservative method is still well founded, but the decompression or the revision of the previous decompression is necessary in case of APP $<60 \mathrm{mmHg}$.

In the past few decades, the consensus definitions were elaborated and following several modifications were published again in 2013 , the diagnostic method was brought to perfection, the therapeutic possibilities were revolutionised and developed to a high-tech level; however, 
the puzzling out of the pathophysiology in its entire depth and as an integer is yet to be achieved $[17,18]$. It is known that the basis of the phenomenon is the co-dependent chain reaction of several physiological processes triggered by the increased intra-abdominal pressure, but the exact mechanism still remains in obscurity [14, 15].

\section{Aims}

The main goal of this chapter was the description of the advantages and disadvantages of the different techniques for monitoring the intra-abdominal pressure. In our works, we wanted to study whether the method of continuous intra-abdominal pressure measurement is feasible within the everyday practice of diagnosing the conditions having increased the intra-abdominal pressure, as well as the ACS. Our aim was not only to validate this new technique but also to bring it to perfection as well.

\section{Comparative study of the traditional and continuous intra-abdominal pressure measurement techniques}

\subsection{Patients of the comparative study of the intra-abdominal pressure measurement techniques}

To carry on the comparative study of the intermittent (traditional) and continuous intraabdominal pressure measurement techniques, 20 patients with acute pancreatitis were involved. The selection of the patients was based on a random nature.

\subsection{Measurement methods}

The intra-abdominal pressure was measured on every patient, in every 6 hours, by both the techniques. To avoid the technical errors, all of the measurements were carried out by the same person. Patients were included into the study following the preliminary oral information and signing of the informed consent forms, for which the patients had the right and possibility of withdrawal made at any time without providing any justification.

\subsubsection{Traditional (intermittent) technique of intra-abdominal pressure measurement}

Prior to our study, protocolised intra-abdominal pressure measurements were never performed at our hospital. Sporadic pressure determinations were performed in one or two clinical centres; however, routine measurements defined in protocols could nowhere be mentioned. In order to carry out the initial pressure measurements, we performed intermittent measurements following the Sugrue technique [8-11]. The patients wore a simple bladder catheter (Foley balloon catheter, 16Fr-20Fr, latex or silicone). During the measurement, the urine collection bag was removed and the bladder was filled with $50 \mathrm{~mL}$ of physiological saline through the lumen of the catheter. In the next step, the lumen of the catheter was connected to a set designed and used for the measurement of the central venous pressure (B. BRAUN Medifix ${ }^{\circledR}$ pressure measurement scale) with or without the insertion of a T-tap. The zero point 
of the scaled measurement tube was designated in the medioaxillary line corresponding to the anterior superior iliac crest. After waiting for 1-2 minutes, at the end of exhalation, the value of IAP could be read off the scale in units of $\mathrm{cmH}_{2} \mathrm{O}$. The values read off should be converted to $\mathrm{mmHg}\left(1 \mathrm{mmHg}=1.36 \mathrm{~cm} \mathrm{H}_{2} \mathrm{O}\right)$. When the measurement was completed, the system and the bladder catheter were disconnected and the latter was connected to a urine collection bag.

\subsubsection{Continuous intra-abdominal pressure measurement}

The technique of continuous intra-abdominal pressure measurement was known from the international literature [13]. With the aid of personal consultations with the Australian working group, which elaborated the procedure (Prof. Zsolt Balogh), we perfected and further developed it, and subsequent to the elaboration of the ward protocol and of further training lectures held for the specialist healthcare workers, we-being the only such institution to do so-introduced it to the everyday routine practice. Taking into consideration the nature of the intra-abdominal pressure being oscillatory even on a daily basis, we considered the use of the continuous intra-abdominal pressure measurement technique to be essential for the everyday routine, as well as during the design of the studies. For the measurements, we used 18 Fr standard three-way bladder catheters (LubriSilTM All-Silicone Foley catheter, C.R. Bard, Inc., Covington, GA, USA). The catheter and the urine collecting bag remained connected for all the time. In order to perform the pressure measurement, the so-called flushing port of the catheter was connected to the insertion of a transducer to a 24-hour bedside monitor. The connection of the flushing port and the transducer was effectuated with a triple tap. The collapse of the bladder was prevented with physiological saline continuously perfused with the speed of $4 \mathrm{~mL} / \mathrm{h}$. The zero point for the fixation of the transducer was established in the plane determined by the axillary median line and the anterior superior iliac crest. After the system was set to zero, the measured data were continuously recorded, in which data could be easily read off from the bedside monitor. The actual IAP value appeared directly in $\mathrm{mmHg}$ and required no further conversion. Pressure values were read off in every hour. The IAP mean value determined on a daily basis was calculated as the average of pressure values recorded in 24 hours $[3,4]$.

\section{Statistical methods}

Between the two methods correlation was expressed using the Lin's concordance correlation coefficient and Bland-Altman's 95\% limits of agreement, overall and also stratified for measurement time. The concordance correlation coefficient evaluates agreement on a continuous measure obtained by two different methods.

The concordance correlation coefficient combines measures of both precision and accuracy to determine how far the observed data deviate from the line of perfect concordance (i.e., the line at $45^{\circ}$ on a square scatterplot). The coefficient increases in value as a function of:

- The nearness of the data's reduced major axis to the line of perfect concordance (the accuracy of the data).

- The tightness of the data about its reduced major axis (the precision of the data). 
With all coefficient estimates exceeding 0.97 and immensely significant, the findings indicate a very high level of concordance between CIAPM and the intermittent method. Bland-Altman's $95 \%$ limits of agreement estimates are within the clinically non-significant ranges of $\pm 2 \mathrm{mmHg}$.

\section{Results}

In order to determine the objectivity of the continuous intra-abdominal pressure measurement, we carried out measurements in patients with normal and elevated intra-abdominal pressures. Significant difference could not be observed between the results of the intermittent measurements and of the new technique. According to the statistical analysis, the concordance correlation coefficient was higher than 0.97 in all cases, which shows a strongly significant agreement between the two different techniques (Figures 1-4). The 95\% limits of agreement of the Bland-Altman method were between the non-significant $\pm 2 \mathrm{mmHg}$ ranges (Figures 5-8).

According to our results, we can summarise that the continuous intra-abdominal pressure monitoring technique is a modern, safe, and accurate method for the IAP monitoring, which provides results immediately, in millimetre of mercury without the need of conversion.

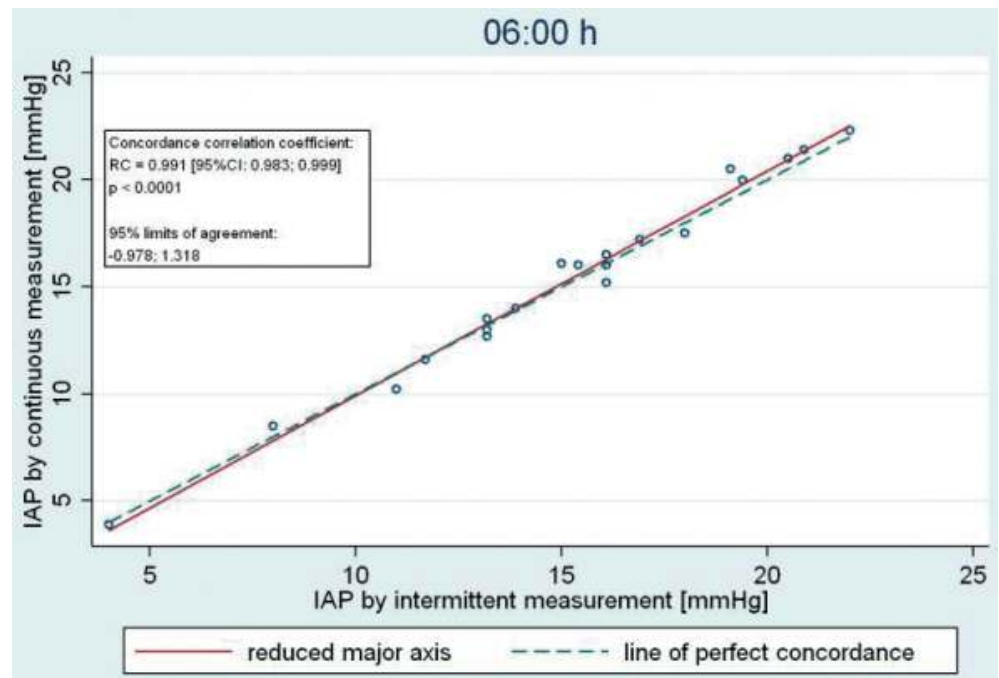

Figure 1. The concordance correlation coefficient was higher than 0.97 in all cases during the measurements carried out at 6.00 hour. 


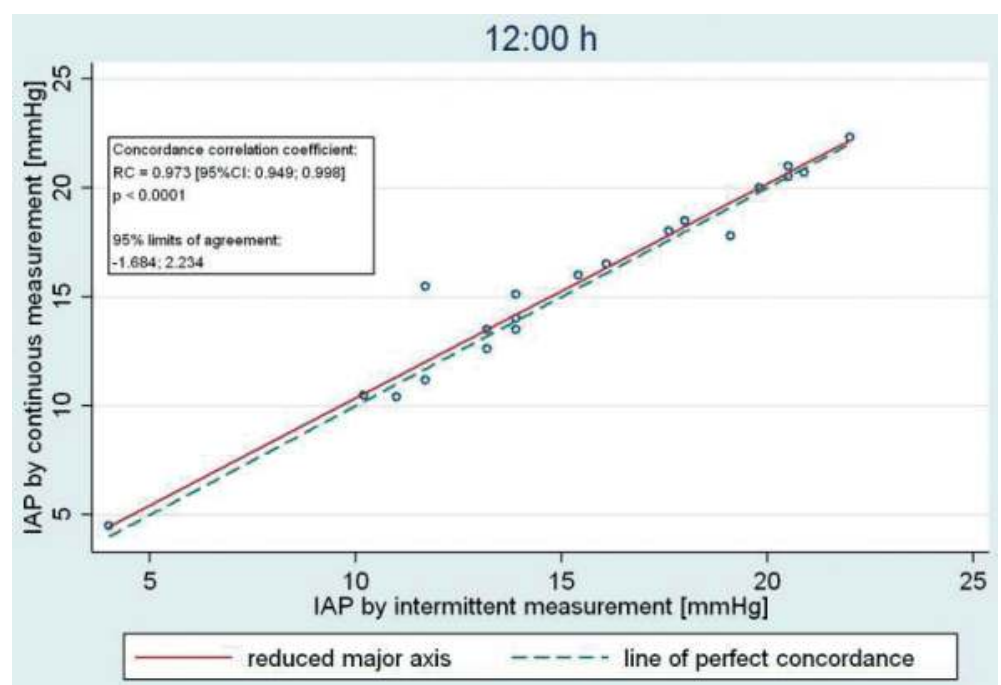

Figure 2. The concordance correlation coefficient was higher than 0.97 in all cases during the measurements carried out at 12.00 hour.

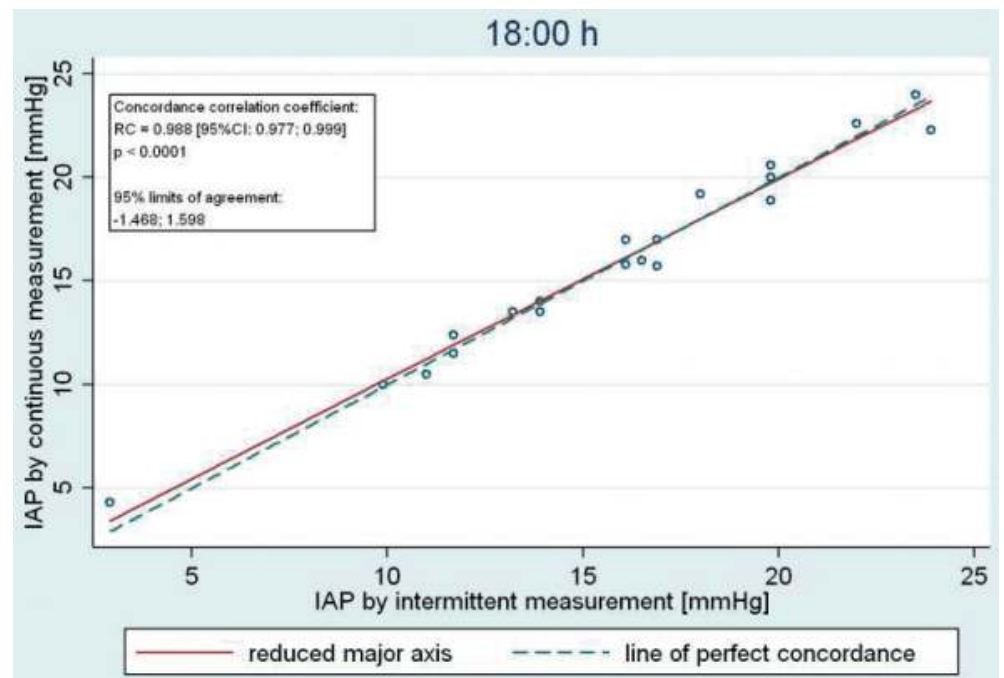

Figure 3. The concordance correlation coefficient was higher than 0.97 in all cases during the measurements carried out at 18.00 hour. 


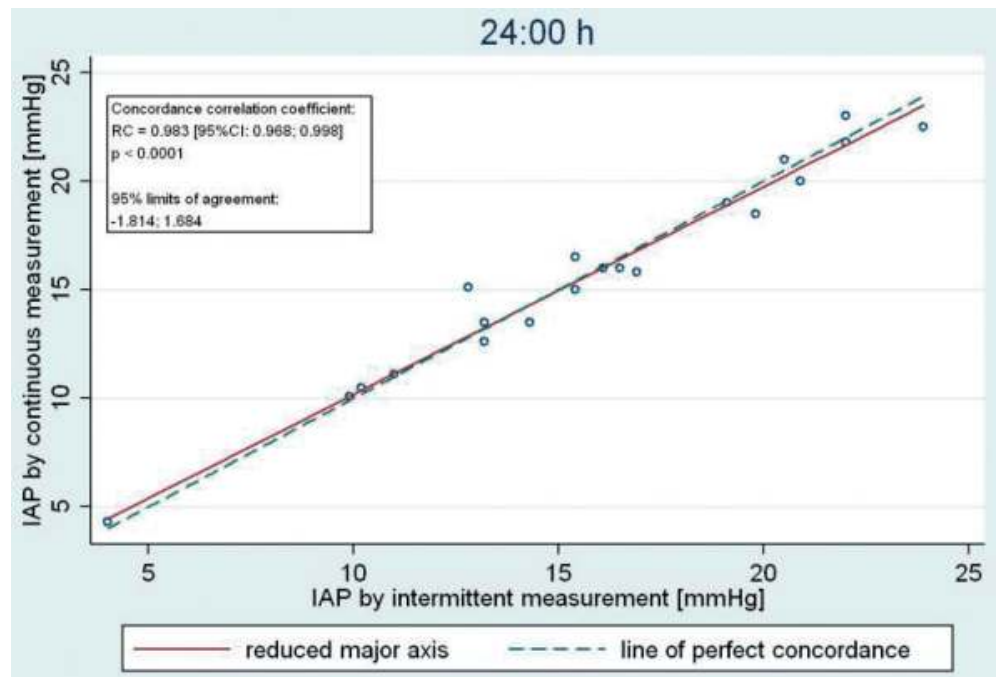

Figure 4. The concordance correlation coefficient was higher than 0.97 in all cases during the measurements carried out at 24.00 hour.

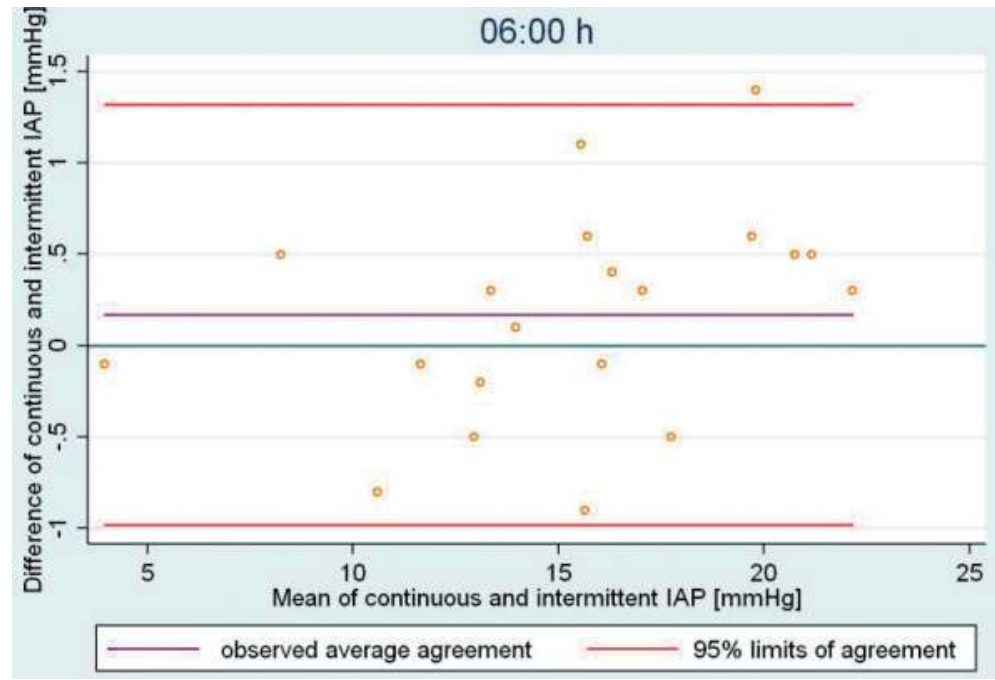

Figure 5. The $95 \%$ limits of agreement of the Bland-Altman method were between the non-significant $\pm 2 \mathrm{mmHg}$ ranges in all cases during the measurements carried out at 6.00 hour. 


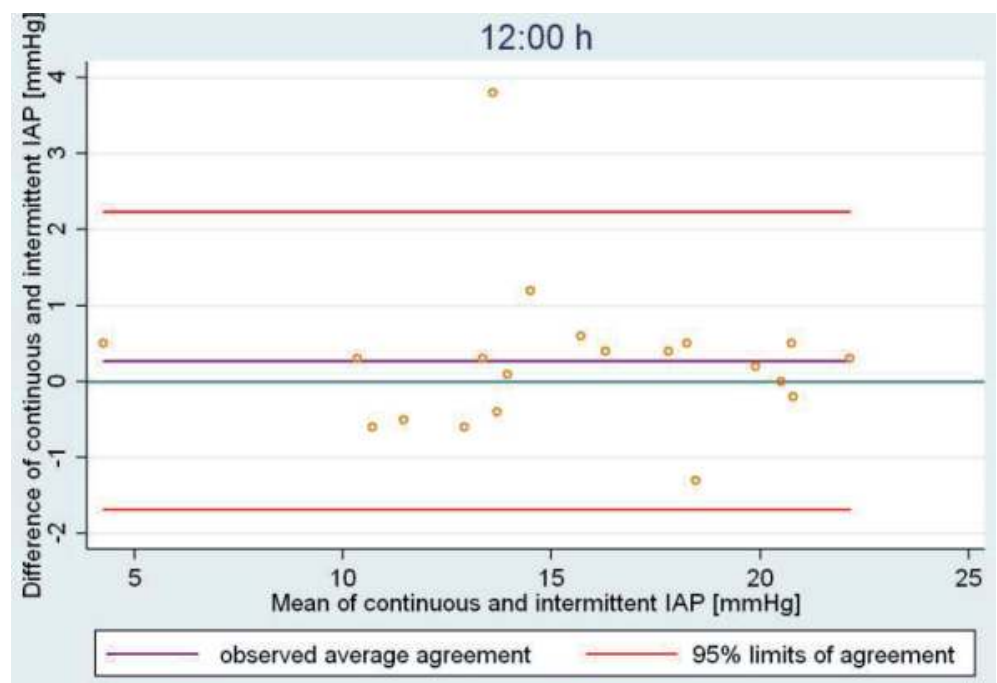

Figure 6. The $95 \%$ limits of agreement of the Bland-Altman method were between the non-significant $\pm 2 \mathrm{mmHg}$ ranges in all cases during the measurements carried out at 12.00 hour.

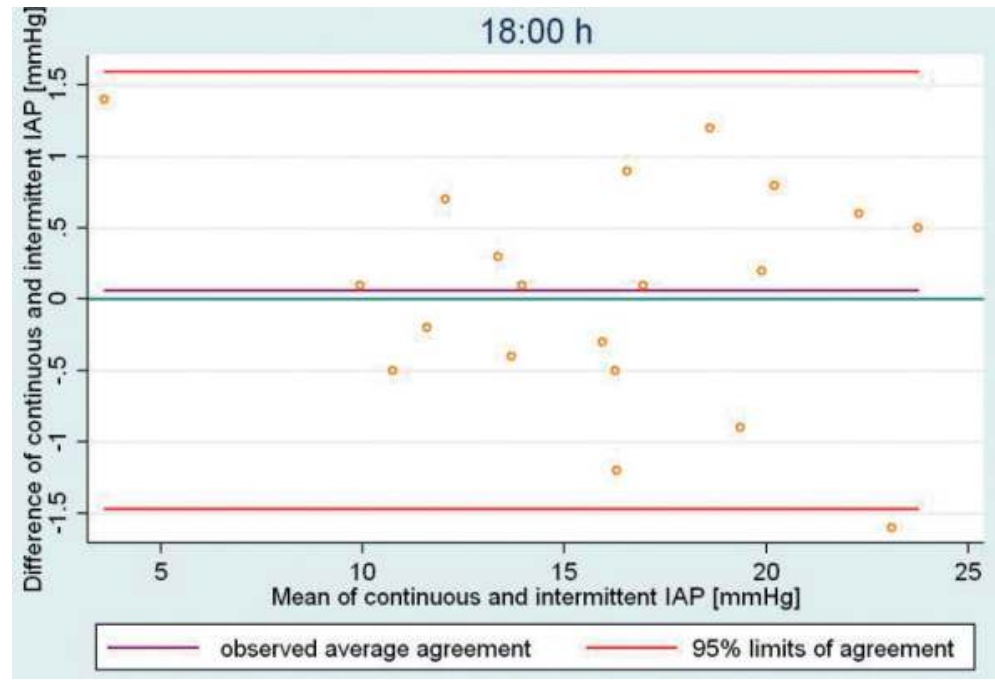

Figure 7. The $95 \%$ limits of agreement of the Bland-Altman method were between the non-significant $\pm 2 \mathrm{mmHg}$ ranges in all cases during the measurements carried out at 18.00 hour. 


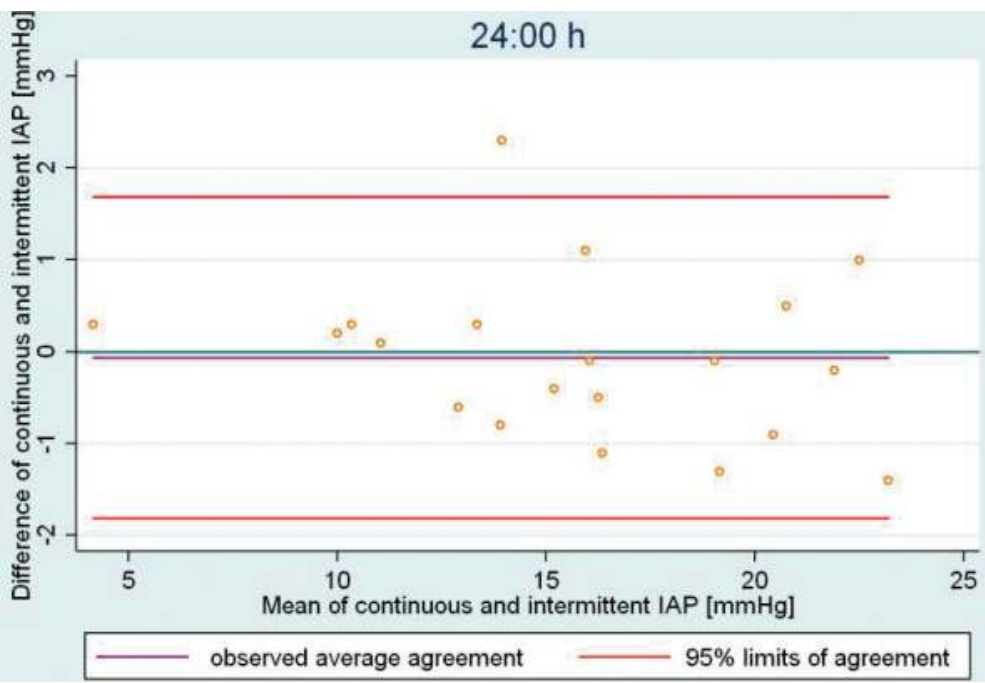

Figure 8 . The $95 \%$ limits of agreement of the Bland-Altman method were between the non-significant $\pm 2 \mathrm{mmHg}$ ranges in all cases during the measurements carried out at 24.00 hour.

\section{Discussion}

Measurement of the intra-abdominal pressure is essential in the differentiated diagnostics of acute abdominal pathologies, in the following of surgery patients being in critical condition, in the prevention of the IAH/ACS, as well as in the monitoring of the already developed syndrome [3-5, 7-13]. The IAP (being within the normal range or increased) is never a constant value but has an oscillatory nature even less than 24 hours [12]. This nature was the main demand to develop a continuous control providing measurement method. The continuous intra-abdominal pressure monitoring technique was first published by Balogh and his working team in 2004 [13]. In order to determine the objectivity of the continuous technique, we carried out measurements in 20 patients, and we verified that the intermittent and continuous measurements are trusted methods of intra-abdominal pressure monitoring without significant differences between them. Following its first construction, the system of CIAPM can be operated without interruption until the next replacement of the bladder catheter (about 7-10 days), thereby eliminating the risk of infection originating from the catheter replacements in the intermittent measurements, as well as the need for extra work and tools.

Summing it up, the continuous intra-abdominal pressure measurement is the "gold standard" of the intra-abdominal pressure monitoring.

Pressure measurement through vesical catheters for the monitoring of the IAP, especially its continuous variant, is an excellent applicable method. Introduction into the daily clinical routine is recommended. 


\section{Author details}

\section{Zsolt Bodnar}

Address all correspondence to: drbozsolt@gmail.com

Letterkenny University Hospital, Letterkenny, Ireland

\section{References}

[1] Balogh Zs, Butcher NE. Compartment syndromes from head to toe. Critical Care Medicine. 2010;38:445-451

[2] Bodnár Zs, Sipka S, Hajdu Z. The abdominal compartment syndrome (ACS) in general surgery. Hepato-Gastroenterology. 2008;55:2033-2038

[3] Bodnár Zs, Ary F, Bulyovszky I, et al. Continuous intra-abdominal pressure measurement technique (CIAP). European Journal of Anaesthesiology. 2006;23(Suppl. 37):197

[4] Bodnár Zs, Sipka S, Szentkereszty Z, et al. The gold standard technique for intra-abdominal pressure monitoring in septic patients: Continuous intra-abdominal pressure monitoring (CIAPM). Inflammation Research. 2007;56(Suppl. 2):213-214

[5] Bodnár Zs. Intra-abdominal pressure measurement. In: Orvostechnika és monitorozás. 1st ed. Boros: Szegedi Tudományegyetem ÁOK Szeged; 2007. p. 53

[6] Kirkpatrick AW, Roberts DJ, De Waele J, et al. Intra-abdominal hypertension and the abdominal compartment syndrome: Updated consensus definitions and clinical practice guidelines from the World Society of the Abdominal Compartment Syndrome. Intensive Care Medicine. 2013;39(7):1190-1206

[7] Ivatury RR. Pressure, perfusion, and compartments: Challenges for the acute care surgeon. The Journal of Trauma and Acute Care Surgery. 2014;76(6):1341-1348

[8] Kron IL, Harman PK, Nolan SP. The measurement of intra-abdominal pressures a criterion for abdominal re-exploration. Annals of Surgery. 1984;199:28-30

[9] Iberti TJ, Lieber CE, Benjamin E. Determination of intra-abdominal pressure using a transurethral bladder catheter: Clinical validation of the technique. Anesthesiology. 1989;70:47-50

[10] Iberti TJ, Kelly KM, Gentili DR, et al. A simple technique to accurately determine intraabdominal pressure. Critical Care Medicine. 1987;15:1140-1142

[11] Sugrue M. Intra-abdominal pressure. Clinical Intensive Care. 1995;6:76-79

[12] Malbrain MLNG. Abdominal pressure in the critically ill: Measurement and clinical relevance. Intensive Care Medicine. 1999;25:1453-1458 
[13] Balogh Zs, Jones F, D'Amours S, et al. Continuous intra-abdominal pressure measurement technique. The American Journal of Surgery. 2004;188:679-684

[14] Bodnár Zs, Keresztes T, Kovács I, et al. Increased serum adenosine and interleukin 10 levels as new laboratory markers of increased intra-abdominal pressure. Langenbeck's Archives of Surgery. 2010;395:969-972

[15] Bodnár Zs, Szentkereszty Z, Hajdu Z, et al. Beneficial effects of theophylline infusions in surgical patients with intra-abdominal hypertension. Langenbeck's Archives of Surgery. 2011;396:793-800

[16] Bodnár Zs, Sipka S, Tidrenczel E, et al. Ten years experience in abdominal compartment syndrome research 2004-2014. Orvosi Hetilap. 2014;155(44):1761-1770

[17] Malbrain MLNG, Cheatham ML, Kirkpatrick A, et al. Results from the International Conference of experts on intra-abdominal hypertension and abdominal compartment syndrome. I. Definitions. Intensive Care Medicine. 2006;32:1722-1732

[18] Cheatham ML, Malbrain MLNG, Kirkpatrick A, et al. Results from the International Conference of experts on intra-abdominal hypertension and abdominal compartment syndrome. II. Recommendations. Intensive Care Medicine. 2007;33:951-962 